\section{Desafíos éticos en la clínica nefrológica}

\section{Ethical Challenges in Nephrological Clinic}

\section{Estimado Sr. Editor.}

Felicitamos a los autores del artículo publicado en la Revista Médica de Chile titulado Problemas ético-clínicos en hemodiálisis crónica: percepción de médicos y enfermeras, por la importante iniciativa y resultados presentados ${ }^{1}$. Es muy interesante observar el interés de los equipos nefrológicos latinoamericanos en abordar los aspectos relacionados al final de la vida de pacientes en diálisis crónica. Estamos de acuerdo con el principal hallazgo de la encuesta de Vukusich, Catoni y col. al referir que el problema ético-clínico percibido con más frecuencia por médicos y enfermeras/os de los centros de diálisis se relaciona a la pertinencia del tratamiento dialítico crónico en pacientes que podrían no beneficiarse del mismo.

El Grupo de ética, calidad de vida y cuidados paliativos de la Sociedad Uruguaya de Nefrología (SUN) realizó una investigación sobre ese tema, publicada en 2014 en la Revista Médica del Uruguay, basada en una encuesta dirigida a nefrólogos ${ }^{2}$.

Nuestro país ha desarrollado su Programa de Diálisis y Trasplante Renal desde XII-1980, creado por ley y financiado por el Fondo Nacional de Recursos, lo que permite el acceso equitativo de la población al tratamiento sustitutivo de la ERC avanzada (TRS). Durante estos 35 años la tasa de incidencia anual en diálisis ha crecido gradualmente, alcanzando a 160 ppm en el período 2011-2015. Como en otros países, este aumento ha sido consecuencia del creciente ingreso de pacientes con $\geq 65$ años en diálisis crónica, de modo tal que ese grupo etario alcanzó una prevalencia del $47,1 \%$ en diálisis en el año 2014. Esta realidad genera nuevos desafíos a los equipos asistenciales, entre los cuales está el abordaje de la comorbilidad múltiple y el frecuente deterioro cognitivo y funcional al que suele evolucionar la población añosa en diálisis. De acuerdo a datos del Registro Uruguayo de Diálisis, no obstante, la mortalidad se ha mantenido estable alrededor de $10 \%$ anual así como la frecuencia de suspensión de diálisis crónica en Uruguay alcanza al 7\% de los fallecidos ${ }^{3}$.

Nuestro Grupo de estudio se propuso conocer las actitudes de los nefrólogos uruguayos respecto a la suspensión o no inclusión de pacientes en plan de diálisis crónica, para lo cual diseñamos una encuesta en base a modelos utilizados antes ${ }^{4,5}$. La encuesta se administró online, luego de una convocatoria por correo electrónico a todos los médicos miembros de la SUN, solicitando que respondieran en forma voluntaria y anónima. Se alcanzó la participación de 63 colegas, o sea $48 \%$ de todos los convocados, de los cuales $82 \%$ tenía más de 45 años de edad, $77 \%$ eran mujeres, y $84 \%$ contaban con más de diez años en la especialidad, siendo considerada ésta como una muestra representativa del grupo nefrológico nacional.

La encuesta planteó tres escenarios clínicos hipotéticos referidos a abandono, inicio o no mantenimiento de diálisis crónica, preguntándose en cada caso ¿Qué haría usted? o ¿Cómo actuaría usted ante esta situación? Al plantear "uno de sus pacientes en diálisis ha evolucionado hasta un estado de demencia severo y permanente", $66 \%$ respondió "probablemente suspendo la diálisis". Ante un paciente competente que solicita suspender el 
plan de diálisis, $86 \%$ procura continuar el tratamiento. $\mathrm{Al}$ solicitar comenzar diálisis crónica en un paciente en estado vegetativo irreversible, $100 \%$ responde "probablemente no comienzo diálisis". Al decidir ante estas situaciones, todos consultarían al paciente y/o familia y a otros técnicos del equipo asistencial.

Asimismo se exploró el conocimiento de los participantes sobre la legislación vigente, sobre su grado de preparación para enfrentar esas decisiones así como sobre qué factores pueden influir en el proceso de adopción de las mismas.

En conclusión, aun cuando solo $38 \%$ de respondedores a la encuesta se sentían preparados para tomar decisiones relacionadas al final de la vida de sus pacientes, la gran mayoría de los nefrólogos estuvieron dispuestos a suspender el plan de diálisis o no incluir en el mismo a pacientes en situaciones consideradas terminales, graves e irreversibles. Las decisiones en esos casos nunca se adoptaban en forma individual sino como equipo multidisciplinario, compartiéndolas con la familia, psicólogo o psiquiatra, otros médicos involucrados y demás integrantes del equipo de salud.

A diferencia de los resultados de Vucusich y col., nuestra encuesta no sugiere existencia de problemas de comunicación entre equipo asistencial y pacientes o familiares, así como no aparece el interés económico de los médicos o instituciones como un factor que afecte la adopción de estas decisiones. De todos modos reconocemos que éstos no fueron objetivos específicamente abordables por nuestro estudio.

Como Vucusich y col., nosotros también hemos concluido que los equipos nefrológicos necesitamos capacitarnos en aspectos de bioética y manejo de cuidados paliativos, así como debemos elaborar guías de práctica clínica a nivel nacional o regional para abordar las decisiones que se vinculan al final de la vida de los pacientes en TRS.

Consideramos importante compartir con colegas de la región estas experiencias de interés común, muy relacionadas al ámbito cultural y lingüístico latinoamericano. Sería deseable mantener una mejor comunicación entre Sociedades Científicas de países vecinos, preocupadas por temas tan sensibles y de alto impacto profesional y humano.
María Nieves Campistrús ${ }^{1}$, Carla Francolino ${ }^{2}$, Juan Dapueto ${ }^{3}$

Grupo de estudio de ética, calidad de vida y cuidados paliativos de la Sociedad Uruguaya de

Nefrología (SUN)

${ }^{1}$ Médico nefróloga, Centro de diálisis

Uruguayana. Montevideo, Uruguay.

${ }^{2}$ Licenciada en Psicología. Profesora Agregada.

Departamento de Psicología Médica de la Facultad de

Medicina de la UDELAR. Montevideo, Uruguay.

${ }^{3}$ Médico internista y Psiquiatra. Profesor titular.

Departamento de Psicología Médica de la Facultad de Medicina de la UDELAR. Montevideo, Uruguay.

\section{Referencias:}

1. Vukusich A, Catoni MI, Salas SP, Valdivieso A, Browne F, Roessler E. Problemas ético-clínicos en hemodiálisis crónica: percepción de médicos y enfermeras. Rev Med Chile 2016; 144: 14-21.

2. Campistrús MN, Francolino C, Schwedt E, García Taibo M, Dapueto J. Encuesta sobre decisiones vinculadas al final de la vida de pacientes que requieren diálisis crónica. Rev Méd Urug 2014; 30 (2): 17-27.

3. González Bedat C, Ferreiro A, Schwedt E, Chifflet L. Registro Uruguayo de Diálisis. Informe Anual 2013-2014. Disponible en: http://nefrouruguay.com. [Consultado el 23 de julio de 2016].

4. Perry E, Swartz R, Smith-Wheelock L, Westbrook J, Buck C. Why is it difficult for staff to discuss advance directives with chronic dialysis patients? J Am Soc Nephrol 1996; 7 (10): 2160-8.

5. Holley JL, Davison SN, Moss AH. Nephrologists' changing practices in reported end-of-life decision-making. Clin J Am Soc Nephrol 2007; 2 (1): 107-11.

Correspondencia a:

Dra. María Nieves Campistrús

Av. Joaquín Suárez 3520. CP 11700. Montevideo. Uruguay.

Tel. 59823363915;

mncampist@gmail.com. 